\title{
Tuberculin responses and risk of pneumonia in immobile elderly patients
}

Katsutoshi Nakayama, Mizue Monma, Takeyasu Fukushima, Takashi Ohrui, Hidetada Sasaki

\begin{abstract}
Background-Delayed type hypersensitivity (DTH) response to tuberculin is an important marker of $T$ helper 1 (Th1) mediated acquired immunity against tuberculosis. Depressed DTH responses to tuberculin are observed in immobile elderly individuals with reduced activities of daily living (ADL) scores. Immobility in older people increases the risk of pneumonia related mortality. The decline in the competence of the immune system might be a reason for the increased susceptibility to infection in the elderly and the depressed DTH response to tuberculin might be a predictor for increased risk of pneumonia.
\end{abstract}

Method-The DTH responses to tuberculin were examined in 49 older patients with limited ADL of similar severity, all of whom had a past history of positive DTH responses to tuberculin. Responses with an induration diameter of $\geqslant 10 \mathrm{~mm}$ were considered positive. Reactivities of Th1 and Th2 lymphocytes taken from the peripheral blood of each subject were also examined and compared between the positive tuberculin responders and the negative tuberculin responders. The rates of pneumonia in these groups were then compared prospectively for two years.

Results-The number of CD4 lymphocytes differed significantly between patients with a positive tuberculin response $\left(n=22\right.$, mean (SE) $\left.1018(118) \times 10^{6} / 1\right)$ and those with a negative response $(n=27$, $\left.666(80) \times 10^{6} / 1, p=0.02\right)$. Likewise, the number of $T h 1$ cells was significantly higher in positive tuberculin responders than in negative responders $(276(5.8) \times$ $10^{6} / 1$ versus 149 (14) $\left.\times 10^{6} / 1, p=0.01\right)$. However, the numbers of $\mathrm{Th} 2$ cells were similar between patients with positive and negative responses $\left(22(3) \times 10^{6} / 1\right.$ versus 19 (3) $\left.\times 10^{6} / 1, p=0.41\right)$. During the follow up period new pneumonia was diagnosed in $18(67 \%)$ of the 27 negative tuberculin responders and in seven $(31 \%)$ of the 22 positive tuberculin responders. According to the Cox regression model, a hazard ratio of developing pneumonia in patients with a negative tuberculin response compared with those with a positive tuberculin response was 2.57 (95\% CI 1.12 to 6.17 , $\mathrm{p}=\mathbf{0 . 0 3 ) \text { . }}$

Conclusion-The diminished DTH response to tuberculin may be a predictor for increased risk of pneumonia in elderly disabled patients.

(Thorax 2000;55:867-869)

Keywords: tuberculin response; pneumonia; $\mathrm{T}$ lymphocyte; geriatrics

After mycobacterial infection $\mathrm{T}$ lymphocytes proliferate and become sensitised. An important marker of $\mathrm{T}$ helper 1 (Th1) mediated acquired immunity against tuberculosis is the development of delayed type hypersensitivity (DTH) responses to tuberculin. ${ }^{1}$ Depressed DTH responses to tuberculin are related to conditions such as old age, cachexia, malnutrition, chronic renal failure, measles infection, and infection with HIV. ${ }^{2}$ This phenomenon, so called cutaneous anergy, is observed in immobile elderly individuals with reduced activities of daily living (ADL) scores. ${ }^{3}$ Furthermore, immobility in older people increases the risk of pneumonia related mortality. ${ }^{4}$ However, it is unknown whether this heightened susceptibility to infection is a reflection of the decline in the competence of the immune system associated with disability. We have therefore studied the association between depressed DTH responses to tuberculin and the risk of pneumonia in older patients with limited ADL by analysing the DTH responses to tuberculin, the responses of Th1 and Th2 lymphocytes, and the rate of pneumonia in these patients.

\section{Methods}

The study was performed in a 220 bed nursing home located in Sendai City, Japan. The level of disability for each patient in the home was coded using the Activities of Daily Living (ADL) index according to Katz and colleagues ${ }^{5}$ in which patients were assigned an ADL score in each of the six major areas of activity (bathing, dressing, toileting, transfer, continence, and feeding). Patients received a score of 1 for each activity if they were fully independent, 2 if they were partially dependent, and 3 if they were completely dependent. Possible total scores ranged from 6 to 18 . A score of 6 indicated that the patient had full function/ independence and a score of 18 indicated that the patient had total disability/dependence. Patients selected to participate in the study were elderly with an ADL index of 13-15, who received part or complete assistance in each area of activity but were fed orally without aspiration and used no indwelling urinary catheter. Forty nine patients (31 women and 18 men) of mean age 79 (1) years (range 66-91) and body mass index $22.0(0.5) \mathrm{kg} / \mathrm{m}^{2}$ 


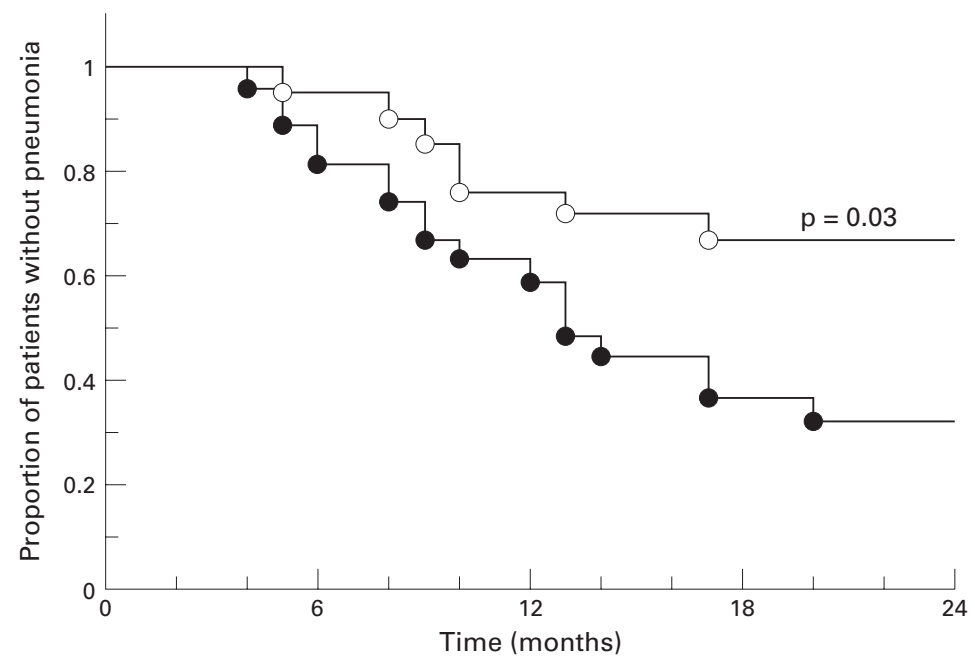

Figure 1 Kaplan-Meier plots of the proportion of patients without pneumonia in patients with a negative tuberculin response ( $n=27$, closed circle) and those with a positive tuberculin response ( $n=22$, open circle).

(range 17.0-29.3) were enrolled. The mean ADL index score was $13.9(0.1)$ points (range 13-15). The causes of disability were cerebral infarction and femoral neck bone fracture. We excluded patients if they were immunocompromised-for example, patients with active malignant disease, those on renal dialysis, those receiving corticosteroid treatment, or with HIV-1 infection. Patients were also excluded from the study if they had swallowing difficulties, a past history of aspiration during feeding, chronic sepsis in pressure sores, venous ulcers, or indwelling urinary catheters. All participants had a past history of a positive DTH response to tuberculin but they were free from clinical symptoms and active lesions of tuberculosis during the two year study. For DTH cutaneous responses to tuberculin upon skin testing, tuberculin purified protein derivative ( 5 tuberculin units) was injected intradermally on the dorsal side of the right forearm. Forty eight hours after injection the diameter of the induration was measured. DTH reactions with a diameter of induration of $\geqslant 10 \mathrm{~mm}$ were estimated as positive. Administration of the skin test was performed by one person who had received sufficient training and reading of the results was confirmed independently by two medical doctors.

To count the number of CD4 lymphocytes, whole blood labelled with a peridinin chlorophyll protein conjugated monoclonal anti-CD4 antibody was analysed with a FACS Calibur flow cytometer. To assess Th1 and Th2 subsets of CD4 lymphocytes, cells positive for antiinterferon- $\gamma$ antibody labelled with fluorescein isothiocyanate and those for anti-interleukin-4 antibody labelled with phycoerythrin under stimulation with $25 \mathrm{ng} / \mathrm{ml}$ phorbol 12myristate 13 -acetate, $1 \mu \mathrm{g} / \mathrm{ml}$ ionomycin, and $10 \mu \mathrm{g} / \mathrm{ml}$ brofeldin were analysed with a Fastimmune cytokine system. This system measured the expression of cytokines inside the cells.

We prospectively compared the rate of pneumonia in the patients with positive tuberculin responses with that in the patients with negative tuberculin responses. All patients were studied for 24 months. Criteria for the diagnosis of pneumonia were a new pulmonary infiltrate seen on the chest radiograph and one of the following features: cough, temperature $>37.8^{\circ} \mathrm{C}$, or subjective dyspnoea.

ANALYSIS OF DATA

Comparisons of age, ADL index score, body mass index, number of CD4 lymphocytes, Th1 cells and Th2 cells between groups were made by the unpaired Student's $t$ test. Underweight patients $\left(\mathrm{BMI}<18.5 \mathrm{~kg} / \mathrm{m}^{2}\right)$ in the two groups were compared by $\chi^{2}$ testing and the rates of pneumonia were compared using the Cox regression model. These analyses were performed using the StatView J-4.5 application program (SAS Institute Inc, North Carolina, USA). A $p$ value of $<0.05$ was considered statistically significant. All values are expressed as mean (SE).

\section{Results}

A tuberculin skin test was performed in 49 patients with limited ADL who were grouped according to positive and negative tuberculin responses. If the diameter of induration 48 hours after injection was $\geqslant 10 \mathrm{~mm}$, the response was considered to be positive. Of 49 disabled patients, 22 of mean age 78 (1) years (range 66-87) and an ADL index score of 13.9 (0.2) points (range 13-15) had a positive tuberculin response; the remaining 27 patients of mean age 80 (1) years (range 66-91) and an ADL score of $14.0(0.2)$ points (range 13-15) had a negative tuberculin response. There were no significant differences in mean age and $\mathrm{ADL}$ index score between these two groups $(p=0.2$ for age, $p=0.6$ for ADL index score). The body mass index of the two groups was also similar $\left(22.2 \quad(0.8) \mathrm{kg} / \mathrm{m}^{2}\right.$ and 21.8 $(0.7) \mathrm{kg} / \mathrm{m}^{2}$ in positive and negative responders, respectively, $p=0.73$ ). Three patients in each group were categorised as being underweight $\left(<18.5 \mathrm{~kg} / \mathrm{m}^{2}\right)$ according to the WHO classification $(13.6 \%$ versus $11.1 \%, \mathrm{p}=0.8)$. The number of CD4 lymphocytes differed significantly between patients with a positive tuberculin response and those with a negative response $\left(1018(118) \times 10^{6} / 1\right.$ versus $666(80) \times$ $\left.10^{6} / 1, \mathrm{p}=0.02\right)$. The mean difference in the number of CD4 lymphocytes between patients with a positive response and those with a negative response was $352 \times 10^{6} / 1\left(95 \%\right.$ CI $73 \times 10^{6}$ to $\left.630 \times 10^{6}\right)$. Likewise, the number of Th 1 cells was significantly higher in patients with a positive tuberculin response than in those with a negative tuberculin response $(276(5.8) \times$ $10^{6} / 1$ versus $\left.149(14) \times 10^{6} / 1, p=0.01\right)$. The mean difference in the number of Th1 cells between these two groups was $128 \times 10^{6} / 1(95 \%$ CI $92 \times 10^{6}$ to $\left.163 \times 10^{6}\right)$. However, the number of Th2 cells was similar in patients with positive and negative responses $(22(3) \times$ $10^{6} / 1$ versus $\left.19(3) \times 10^{6} / 1, \mathrm{p}=0.41\right)$. The mean difference in the number of Th2 cells between the two groups was $3.3 \times 10^{6} / 1(95 \%$ CI $-4.3 \times 10^{6}$ to $\left.10.8 \times 10^{6}\right)$. During the follow up period new pneumonia was diagnosed in 18 $(67 \%)$ of the 27 patients who had a negative 
tuberculin response and in seven $(31 \%)$ of the 22 patients who had a positive tuberculin response. According to the Cox regression model, a hazard ratio of developing pneumonia in patients who had a negative tuberculin response compared with those who had a positive response was 2.57 (95\% CI 1.12 to 6.17 , $\mathrm{p}=0.03$; fig 1 ).

\section{Discussion}

Almost all healthy Japanese people over 60 years have positive tuberculin responses at the present time because they have been living in a period with the highest prevalence of tuberculosis. ${ }^{1}$ In fact, all participants in the present experiment had a history of a positive DTH response to tuberculin. However, some patients with limited ADL showed diminished DTH responses to tuberculin, as we have reported elsewhere. We have shown that an impaired DTH response in patients with limited ADL is associated with a decrease in the number of Th1 cells, but not of Th2 cells. Furthermore, we have shown that disabled patients with a negative DTH response have a higher risk of pneumonia than those with a positive DTH response.

Although developed countries including Japan have made remarkable progress in tuberculosis control during the past several decades as a result of socioeconomic improvements, removal of infectious patients from the community, effective vaccine programmes, and the wide availability of antituberculosis drugs, the notifications of patients with tuberculosis have recently started to increase in many countries. ${ }^{6}$ It was also reported that there were an estimated 7.96 million new cases of tuberculo- sis and 16.2 million existing cases in 212 countries in 1997, and the global prevalence of Mycobacterium tuberculosis infection was calculated as 1.86 billion people (32\% of world population) in that year. ${ }^{7}$ The DTH response to tuberculin may therefore be useful for estimating both the presence of infection and the immunological reactivity of individuals who have close contact with Mycobacterium tuberculosis such as Japanese elderly people. Using the DTH response to tuberculin, which is presumed to be dependent on Th1 cells, we suggest that disabled patients with a negative tuberculin response are more likely to suffer pneumonia than those with a positive tuberculin response.

Frail elderly people are likely to suffer pneumonia. ${ }^{4}$ The present simple measurement may help us to identify those with reduced immunity and to give careful management to prevent the development of pneumonia.

1 Shirakawa T, Enomoto T, Shimazu SI, et al. The inverse association between tuberculin responses and atopic disorder. Science 1997;275:77-9.

2 Pesanti EL. The negative tuberculin test. Tuberculin, HIV, and anergy panels. Am $\mathcal{F}$ Respir Crit Care Med 1994;149: 1699-709.

3 Fukushima T, Nakayama K, Monma M, et al. Depression of $\mathrm{T}$ helper-1 and tuberculin responses in older bed-bound patients. F Am Geriatr Soc 1999;47:259-60.

4 Salive MD, Satterfield S, Pstfeld AM, et al. Disability and cognitive impairment are risk factors for pneumoniacognitive impairment are risk factors for pneumonia-
related mortality in older adults. Public Health Rep 1993;108:314-22.

$5 \mathrm{Katz}$ S, Downs TD, Cash HR, et al. Progress in development of the index of ADL. Gerontologist 1970;10: 20-30.

6 Sbarbaro JA. "Elimination" of tuberculosis or of tuberculosis control programs. Bull Int Union Tuberc Lung Dis 1990; 65:47-8.

7 Dye C, Scheele S, Dolin P, et al. Global burden of tubercucountry. $7 A M A$ 1999;282:677-86. 